\title{
BMJ Open Cancer and suicidal ideation and behaviours: protocol for a systematic review and meta-analysis
}

\author{
Raffaella Calati, ${ }^{1,2,3}$ Fang Fang, ${ }^{4}$ Elizabeth Mostofsky, ${ }^{5,6}$ Qing Shen, ${ }^{4}$ \\ Valentina Elisabetta Di Mattei, ${ }^{7,8}$ Jesus Garcia-Foncillas, ${ }^{9}$ Enrique Baca-Garcia, ${ }^{10,11}$ \\ Andrea Cipriani, ${ }^{12}$ Philippe Courtet $^{1,2,3}$
}

To cite: Calati R, Fang F, Mostofsky E, et al. Cancer and suicidal ideation and behaviours protocol for a systematic review and meta-analysis. BMJ Open 2018;8:e020463. doi:10.1136/ bmjopen-2017-020463

- Prepublication history and additional material for this paper are available online. To view these files, please visit the journal online (bmjopen-2017-020463).

Received 6 November 2017 Revised 17 May 2018 Accepted 24 May 2018

\section{Check for updates}

(c) Author(s) (or their employer(s)) 2018. Re-use permitted under CC BY-NC. No commercial re-use. See rights and permissions. Published by BMJ.

For numbered affiliations see end of article.

Correspondence to

Raffaella Calati;

raffaella.calati@gmail.com

\section{ABSTRACT}

Introduction Prevalence of suicidal ideation (SI) and behaviours are higher among patients with cancer than general population. No systematic review/meta-analysis investigated this topic; therefore, our aim will be to assess the relationship between cancer and SI and behaviours. Methods We will search PubMed/MEDLINE, EMBASE, SCOPUS, Web of Science, PsycINF0 and Cochrane Library databases from their inception until 30 June 2018. Casecontrol and cohort studies focused on the association between cancer (any type) and suicidal outcomes (suicide, suicide attempt and SI) will be included. Two team members will independently: $(A)$ perform the selection of the included studies and data extraction, with the supervision of a third member in case of discrepancies and (B) assess each study with: (1) Newcastle-Ottawa Scale (NOS); (2) Strengthening the Reporting of Observational Studies in Epidemiology (STROBE) statement; (3) Grading of Recommendations Assessment, Development and Evaluation (GRADE). We will conduct a random-effects meta-analysis. Individual and pooled ORs and associated $95 \% \mathrm{Cls}$ will be calculated as well as between-study heterogeneity. We will examine the potential for publication bias. If possible, we will explore reasons for potential between-study heterogeneity.

Ethics and dissemination This study does not require ethical approval. The study will be submitted to a peerreviewed journal, will be publicly disseminated and will be the topic of research presentations.

PROSPERO registration number CRD42017072482.

\section{INTRODUCTION}

In 2015, cancer was the second leading cause of death worldwide, ${ }^{1}$ and suicide was the 17 th cause of death and the second among 15-29 year olds. ${ }^{2}$ Cancer might also lead to suicide: prior studies have shown that suicide incidence among patients with cancer is almost double compared with the general population. ${ }^{3-5}$ Moreover, patients with cancer were found to be particularly prone to suicidal ideation (SI), especially in the case of advanced cancer, ${ }^{4}$ and suicide attempts (SAs) ${ }^{56}$ as well. However, little is known about: (1) the timing of suicide risk

\section{Strengths and limitations of this study}

- This will be the first systematic review and meta-analysis on the topic.

- Implications of our future findings might provide evidence to the improvement of policies on suicidal symptom screening by healthcare professionals working in oncology.

- The findings could be limited to determine the timing of suicide risk after a cancer diagnosis and the specific cancer sites associated with suicide due to the less investigation from previous studies.

following a cancer diagnosis; (2) whether findings are consistent across different populations and (3) the shared risk factors.

1. Concerning the timing of risk following the cancer diagnosis, one study reported that standardised mortality ratio (SMR: the ratio of observed deaths in the study group to expected deaths in the general population) was 3.09 for men and 2.18 for women within the first 5 months after diagnosis; during 12-23 months after cancer diagnosis, SMR decreased but remained elevated to 1.57 and 1.72 , respectively. ${ }^{7}$ In another study, risk of suicide was highest during the first 3 months for men, while highest from 3 to 12 months for women. ${ }^{8}$ Such difference can be explained by different prognosis, in particular to poor prognosis in the first peak (3 months) and to moderate-good prognosis in the second risk increase (1214 months $)^{9}$; the last peak of risk elevation has been explained by the possible cancer recurrence after the failure of treatment. Moreover, patients with prostate cancer seem to be particularly at risk of suicide after 15 years since the cancer diagnosis. ${ }^{1011}$ This finding has been tentatively interpreted with late-onset metastases and/or symptoms, long-term urinary incontinence and erectile dysfunctions. ${ }^{11}$ Hence, suicide risk 
may shift, from being related to psychological effects linked to the diagnosis in the short term, to being associated to cancer-related sequelae, side effects of cancer treatment, fear of cancer recurrence and further ageing-related factors in the long term. ${ }^{10}$

2. Concerning consistency across populations, gender, age, income and regional differences have been reported. Male patients with cancer seem to have a higher suicide risk than females. ${ }^{5}$ The highest suicide risk was reported among people aged $40-46$ and over 60 years. ${ }^{5}$ Among patients with cancer, higher income was associated with less SI. ${ }^{12}$ Regional differences were also found: the association between cancer and suicide was found to be stronger in Eastern European nations. ${ }^{13}$ Authors hypothesised that this association could be related to indicators of social disintegration (such as divorce, abortion and illegitimacy rates, and urbanicity) but only in less developed regions (eg, Ukraine vs USA). ${ }^{14} 15$ Accordingly, living alone, further indicator of social disintegration, is associated with suicide. ${ }^{16}$

3. Pertaining risk factors, SI and behaviours seem to be linked to the same risk factors in patients with cancer and in the general population: male gender, older age, depression, hopelessness, pain and poor physical functioning. In particular, depressive disorders (including major depression, minor depression and other forms of depressive conditions, such as demoralisation) affect up to $60 \%$ of patients with cancer. ${ }^{17}$ However, depression in these patients could not be recognised because it could be hard to distinguish depressive symptoms from some cancer treatment-related and cancer disease-related symptoms, such as fatigue. ${ }^{18}$ Further risk factors for suicide among individuals diagnosed with cancer include the already described time since diagnosis, cancer site, advanced stage of cancer at diagnosis and poor prognosis. ${ }^{45}$ The majority of studies did not examine specific cancer types and some pooled together more severe forms of cancer, such as oesophagus, liver, pancreas and lung cancers. ${ }^{3}$ Although disease with advanced stage and poor prognosis seem to be linked to higher suicide risk, increased suicide risk was also observed in patients with low-risk prostate cancer. ${ }^{19}$

In particular, shared risk factors (eg, smoking, alcohol consume and air pollution) might lead to spurious associations between cancer and suicide conditions. ${ }^{20-23}$ The evaluation of other factors, such as pain ${ }^{24-26}$ and social support, ${ }^{2728}$ may also help in the identification of new targeted prevention strategies.

To our knowledge, no previous systematic review and meta-analysis investigated the association between a cancer diagnosis and suicide risk. Thus, the aim of this study is to assess the risk of SIs and behaviours after a cancer diagnosis. Secondary aim of this study will be to specifically address the knowledge gaps in the previous literature, focusing on the timing of suicide risk and controlling for all the possible confounding factors. We will focus not only on suicide but also on SI and SAs since they are risk factors for suicide. ${ }^{29}$ Our future findings might shed further light on the improvement of on-time suicidal symptom screening among patients with cancer. For instance, healthcare professionals working in oncology should receive specific training to identify and treat depression and SI and behaviours.

\section{OBJECTIVES}

To investigate the association between cancer diagnosis and

1a. Suicide,

1b. SA,

1c. SI.

These analyses will be performed controlling for confounding factors when possible.

\section{METHODS}

The present protocol has been registered in PROSPERO and is reported in accordance with the Preferred Reporting Items for Systematic Review and Meta-Analysis Protocols statement. ${ }^{30}$

The meta-analysis will be reported in accordance with the reporting checklist proposed by the Meta-analysis of Observational Studies in Epidemiology group. ${ }^{31}$

\section{Types of studies}

Studies will be included if: they are case-control and both prospective and retrospective cohort studies of any length of follow-up; they investigate the association between cancer diagnosis and subsequent SI and behaviours; they report data on cancer diagnoses and suicidal outcomes; they focus on any type of study population. If available, we will use estimates of the association (ORs, risk ratios or rate ratios) and 95\% CIs that have accounted for potential confounders; otherwise, we will include data on the number of cases and non-cases with and without cancer diagnoses to calculate crude estimates of the association, and we will conduct sensitivity analyses restricted to adjusted estimates.

Studies will be excluded if: they are not written in English; they focus on patients with cancer only or suicidal patients only; they pool different suicidal outcomes together (eg, suicide and SA) and separate data are not available after having contacted the authors; they rely on self-reported cancer diagnoses only; they are randomised controlled trials, because we aimed to exclude the potential bias of treatment interventions; they are cross-sectional studies, because we focus on suicide risk after cancer diagnosis.

\section{Types of participants}

We will include studies of subjects regardless of age, sex or ethnicity who were participants in observational studies (case-control and cohort) from inpatient, outpatient or mixed community settings with any cancer diagnosis.

\section{Cancer}

We will include studies that examine the impact of a diagnosis of any malignant neoplasm (International 
Classification of Diseases (ICD)-9: 140-209; ICD-10: C00C97). In sensitivity analyses, we will consider site-specific cancers if sufficient data are available.

\section{Suicidal outcomes}

We will refer to established nomenclature. ${ }^{29}$ We will separately consider all the suicidal events as reported by the original study authors: suicide (self-inflicted death), SA (self-inflicted potentially injurious behaviour with a non-fatal outcome and with the intention to die) and SI (thinking of dying of suicide). If possible, we will consider the distinction between passive SI (eg, thoughts of being better off dead) and active SI as well. We will include deliberate self-harm only if it is possible to distinguish non-suicidal self-injury (NSSI) and SA (NSSI refers to an intentional act of causing physical injury to oneself without wanting to die). As outcomes, we will consider the number of events (suicide and SA) and any standardised rating scale for assessing the presence of SI; it could be an item as for Montgomery-Åsberg Depression Rating Scale ${ }^{32}$ or Hamilton Rating Scale for Depression, ${ }^{33}$ or a specific scale about suicide, like the Columbia-Suicide Severity Rating Scale. ${ }^{34}$ We will consider SI as presence versus absence. If only mean score measures of SI are shown in an article, we will contact the authors.

\section{Search methods for the identification of studies Electronic searches}

We will search PubMed/MEDLINE, EMBASE, SCOPUS, Web of Science, PsycINFO and Cochrane Library, from their inception until 30 June 2018, to identify cohort and case-control studies reporting the association between cancer and suicidal outcomes. Combinations of Medical Subject Headings terms will be: [cancer* OR tumor* OR carcinoma* OR neoplas* OR oncolog* OR metastas* OR malign*] AND [suicid* OR self-harm* OR self harm OR self-poisoning OR self poisoning] (see online supplementary document 1 ).

\section{Reference lists}

The reference lists of all the included studies, relevant papers and previous systematic reviews will be also handsearched for the identification of additional studies.

\section{Data collection}

Selection of studies

Two authors (RC and QS) will independently check the titles and abstracts of all the references generated by the search strategies to decide if they meet the inclusion criteria. All studies potentially eligible for inclusion will be added to the preliminary list, and their full-text articles will be retrieved. The two authors will then assess all full-texts to verify if they meet the inclusion criteria. If the authors disagree, the final decision will be made by consensus with the involvement of FF, EM or AC.

\section{Data extraction and management}

Using a standardised data extraction sheet, RC and QS will independently extract data from the included studies. Any disagreement will be discussed with a third member of the review team (FF, EM or AC), and decisions will be documented. In the case of missing information concerning the outcomes of interest, we will directly contact study authors up to three times to obtain additional information.

The following data will be extracted from all studies meeting the inclusion criteria: country, study design, year, sample size and type, period of assessment, hazard period (ie, the assessed time period after the cancer diagnosis), suicide outcomes, percentage of males, age, ethnicity, cancer type, main results, list of confounders included in design and analysis, crude numbers and measure of association (ORs) and 95\% CIs, and data source.

When available, we will consider both raw data and the models adjusted for covariates. If a study conducts one analysis of men and one of women, those will be included as two separate studies.

In the case of studies published on the same data source, we will use the most recently published results or the largest sample size. To avoid the risk of overlapping studies for each study, we will extract: (1) the names of the authors and (2) the names of the databases/studies (data source) and we will check for duplicates; then, in the case of doubts, we will contact the authors.

\section{Assessment of quality, strength of reporting and certainty of evidence in included studies}

Two authors (RC and VEDM) will independently assess the quality of the studies using the Newcastle-Ottawa Scale $(\mathrm{NOS})^{35}$ and the strength of reporting of studies according to the Strengthening the Reporting of Observational Studies in Epidemiology (STROBE) statement. ${ }^{36}$ The NOS assesses with eight questions the quality of case-control and cohort studies in three broad categories: (1) patient selection; (2) comparability of study groups; (3) assessment of the outcome. The STROBE statement consists of a 22-item checklist for the assessment of the strengths and weaknesses of each section of a single study (title, abstract, introduction, methods, results and discussion). The Grading of Recommendations Assessment, Development and Evaluation (GRADE) methodology for evaluating the certainty of evidence for each outcome will be used as well. ${ }^{37}$ The certainty of evidence will be classified as high (further research is very unlikely to change our confidence in the estimate of effect), moderate (further research is likely to have an important impact on our confidence in the estimate of effect and may change the estimate), low (further research is very likely to have an important impact on our confidence in the estimate of effect and is likely to change the estimate) or very low (any estimate of effect is very uncertain).

\section{Data analysis}

Main analyses

We will calculate pooled ORs and 95\% CIs. We will assess heterogeneity with the $\chi^{2}$ goodness of fit and $\mathrm{I}^{2}$ statistics. Concerning $\mathrm{I}^{2}$, we will consider Cochrane 
recommendations. ${ }^{38}$ We will consider statistically significant a $p$ value $<0.05$ (presence of heterogeneity). In the case of the presence of heterogeneity, we will perform sensitivity analyses and metaregressions when possible.

We will use a random-effects model since we hypothesise that the effect will be similar but not identical across studies. A funnel plot will be created to reveal the preferential publication of statistically significant results. Tests for funnel plot asymmetry will be used in the presence of at least 10 studies. The Egger's test will be also used to evaluate the funnel plot asymmetry. ${ }^{39}$

\section{Additional analyses}

We would like to calculate the pooled prevalence for each suicidal outcome in subjects with and without cancer. Moreover, if there is a sufficient number of studies for each suicidal outcome, we will investigate potential sources of heterogeneity using metaregression models, and we will perform subgroup analyses by sociodemographic (eg, age, sex, ethnicity, socioeconomic status, social support) and clinical characteristics (eg, smoking and other substance use, physical disorders, pain, cancer type, cancer stage $(0, \mathrm{I}$, II, III and IV), early and late suicide (early suicide: suicide during the first year after the cancer diagnosis) ) and by study design (case-control and cohort). If possible, we will also compare subjects died by suicide to subjects died by accidental causes (myocardial infarction, road traffic and motor vehicle accidents, homicide, domestic and industrial accidents or any other external cause) as a secondary outcome. If sufficient data are available, we will perform a subgroup analysis on adult cancer survivors only.

\section{Further potential points of discussion}

Even if it might be outside of the scope of this study, considering the constant increase of the number of patients requesting Euthanasia or Assisted Suicide (EAS) in countries where this procedure is allowed ${ }^{40}$ is mandatory. So, we will also take into account this issue, trying to differentiate between patients with cancer with a terminal condition and patient with a better prognosis requesting EAS.

\section{Software}

All analyses will be conducted in Stata V. 14.

\section{Patient and public involvement}

Patients and/or public were not involved.

\section{Strengths and limitations of this study}

To our knowledge, this will be the first systematic review and meta-analysis investigating the relationship between cancer and SI and behaviours. The implications of our future findings might provide evidence to the improvement of policies on suicidal symptom screening by healthcare professionals working in oncology.

Limitations mainly included the lack of investigation on specific topics: the number of studies investigating the timing of suicide risk following a cancer diagnosis, and specific cancer sites associated with suicide could be limited; also, it could be difficult to distinguish depressive symptoms from some cancer treatment-related and cancer disease-related symptoms.

\section{Ethics and dissemination}

No ethical approval is required to perform this study. We will publish results in a peer-reviewed scientific journal and data set will be made freely available.

\section{Author affiliations}

${ }^{1}$ Department of Psychiatric Emergency \& Post-Acute Care, Lapeyronie Hospital, CHU Montpellier, Montpellier, France

${ }^{2}$ FondaMental Foundation, Créteil, France

${ }^{3}$ INSERM, University of Montpellier, Neuropsychiatry: Epidemiological and Clinical Research, Montpellier, France

${ }^{4}$ Department of Medical Epidemiology and Biostatistics, Karolinska Institutet, Stockholm, Sweden

${ }^{5}$ Department of Epidemiology, Harvard School of Public Health, Boston, Massachusetts, USA

${ }^{6}$ Cardiovascular Epidemiology Research Unit, Department of Medicine, Beth Israel Deaconess Medical Center, Harvard Medical School, Boston, Massachusetts, UK

${ }^{7}$ Vita-Salute San Raffaele University, Milan, Italy

${ }^{8}$ Clinical and Health Psychology Unit, IRCCS San Raffaele Hospital, Milan, Italy

${ }^{9}$ OncoHealth Institute, Department of Oncology, Translational Oncology Division, Health Research Institute University Hospital "Fundacion Jimenez Diaz",

Autonomous University of Madrid, Madrid, Spain

${ }^{10}$ Department of Psychiatry, IIS-Jimenez Diaz Foundation, CIBERSAM, Madrid, Spain

${ }^{11}$ Universidad Católica del Maule, Talca, Chile

${ }^{12}$ Department of Psychiatry, University of Oxford, Oxford, UK

Contributors $\mathrm{RC}$ wrote the first draft of the protocol and subsequently incorporated the suggested revisions. FF, EM, QS and AC revised each section of the protocol. VEDM, JG-F and EB-G globally revised the writing. PC supervised the entire project.

Funding This work is partly supported by the Swedish Cancer Society (grant no: CAN 2014/417), Swedish Research Council for Health, Working Life, and Welfare (grant no: 2017-00531), Karolinska Institutet (Senior Researcher Award and Strategic Research Area in Epidemiology Award).

Competing interests $\mathrm{RC}$ received a grant from FondaMental Foundation, Créteil, France (2015-2016). PC received research grants from Servier and fees for presentations at congresses or participation in scientific boards from Janssen, Lundbeck, Otsuka, Servier.

Patient consent Not required.

Provenance and peer review Not commissioned; externally peer reviewed.

Open access This is an open access article distributed in accordance with the Creative Commons Attribution Non Commercial (CC BY-NC 4.0) license, which permits others to distribute, remix, adapt, build upon this work non-commercially, and license their derivative works on different terms, provided the original work is properly cited, appropriate credit is given, any changes made indicated, and the use is non-commercial. See: http://creativecommons.org/licenses/by-nc/4.0/.

\section{REFERENCES}

1. Fitzmaurice C, Allen C, Barber RM, et al. Global Burden of Disease Cancer Collaboration. Global, Regional, and National Cancer Incidence, Mortality, Years of Life Lost, Years Lived With Disability, and Disability-Adjusted Life-years for 32 Cancer Groups, 1990 to 2015: A Systematic Analysis for the Global Burden of Disease Study. JAMA Oncol 2017;3:524-48.

2. World Health Organization. 2017 http://www.who.int/mental_health/ prevention/suicide/suicideprevent/en/ (cited 201715 Sep 2018).

3. Fang F, Fall K, Mittleman MA, et al. Suicide and cardiovascular death after a cancer diagnosis. N Engl J Med 2012;366:1310-8.

4. Anguiano L, Mayer DK, Piven ML, et al. A literature review of suicide in cancer patients. Cancer Nurs 2012;35:E14-26.

5. Spoletini I, Gianni W, Caltagirone C, et al. Suicide and cancer: where do we go from here? Crit Rev Oncol Hematol 2011;78:206-19.

6. Druss B, Pincus H. Suicidal ideation and suicide attempts in general medical illnesses. Arch Intern Med 2000;160:1522. 
7. Hem E, Loge $\mathrm{JH}$, Haldorsen $\mathrm{T}$, et al. Suicide risk in cancer patients from 1960 to 1999. J Clin Oncol 2004;22:4209-16.

8. Yousaf U, Christensen ML, Engholm G, et al. Suicides among Danish cancer patients 1971-1999. Br J Cancer 2005;92:995-1000.

9. Dormer NR, McCaul KA, Kristjanson LJ. Risk of suicide in cancer patients in Western Australia, 1981-2002. Med J Aust 2008;188:140-3.

10. Klaassen Z, Arora K, Wilson SN, et al. Decreasing suicide risk among patients with prostate cancer: Implications for depression, erectile dysfunction, and suicidal ideation screening. Urol Oncol 2018;36.

11. Klaassen Z, Jen RP, DiBianco JM, et al. Factors associated with suicide in patients with genitourinary malignancies. Cancer 2015;121:1864-72.

12. Lee SJ, Park JH, Park BY, et al. Depression and suicide ideas of cancer patients and influencing factors in South Korea. Asian Pac J Cancer Prev 2014;15:2945-50.

13. Kondrichin SV, Lester D. Cancer and suicide. Percept Mot Skills 2001;92:468.

14. Kondrichin SV, Lester D. Suicide in the Ukraine. Crisis 2002;23:32-3

15. Kondrichin SV, Lester D. Correlates of regional suicide and cancer mortality rates. Percept Mot Skills 2001;93:338.

16. Schneider B, Lukaschek K, Baumert J, et al. Living alone, obesity, and smoking increase risk for suicide independently of depressive mood findings from the population-based MONICA/KORA Augsburg cohort study. J Affect Disord 2014:152-154:416-21.

17. Caruso R, GiuliaNanni M, Riba MB, et al. Depressive Spectrum Disorders in Cancer: Diagnostic Issues and Intervention. A Critical Review. Curr Psychiatry Rep 2017;19:33.

18. Ullman K. Reducing Risk of Suicide in Cancer Patients. J Natl Cancer Inst 2017;109:3-5.

19. Carlsson S, Sandin F, Fall K, et al. Risk of suicide in men with lowrisk prostate cancer. Eur J Cancer 2013;49:1588-99.

20. Huxley RR, Ansary-Moghaddam A, Clifton P, et al. The impact of dietary and lifestyle risk factors on risk of colorectal cancer: a quantitative overview of the epidemiological evidence. Int $J$ Cancer 2009;125:171-80.

21. Hamra GB, Guha N, Cohen A, et al. Outdoor particulate matter exposure and lung cancer: a systematic review and meta-analysis. Environ Health Perspect 2014;122:906-11.

22. Sinyor M, Tse R, Pirkis J. Global trends in suicide epidemiology. Curr Opin Psychiatry 2017;30:1-6.

23. Xuan Z, Naimi TS, Kaplan MS, et al. Alcohol Policies and Suicide: A Review of the Literature. Alcohol Clin Exp Res 2016;40:2043-55.

24. Calati R, Laglaoui Bakhiyi C, Artero S, et al. The impact of physical pain on suicidal thoughts and behaviors: Meta-analyses. J Psychiatr Res 2015;71:16-32.
25. Choi NG, DiNitto DM, Marti CN, et al. Physical Health Problems as a Late-Life Suicide Precipitant: Examination of Coroner/Medica Examiner and Law Enforcement Reports. Gerontologist 2017 (Epub 2017/09/29)

26. Zhong BL, Li SH, Lv SY, et al. Suicidal ideation among Chinese cancer inpatients of general hospitals: prevalence and correlates. Oncotarget 2017;8:25141-25150.

27. Geng Z, Ogbolu Y, Wang J, et al. Gauging the Effects of Self-efficacy, Social Support, and Coping Style on Self-management Behaviors in Chinese Cancer Survivors. Cancer Nurs 2018:1.

28. Gariépy G, Honkaniemi H, Quesnel-Vallée A. Social support and protection from depression: systematic review of current findings in Western countries. Br J Psychiatry 2016;209:284-93.

29. Turecki G, Brent DA. Suicide and suicidal behaviour. Lancet 2016;387:1227-39.

30. Shamseer L, Moher D, Clarke M, et al. Preferred reporting items for systematic review and meta-analysis protocols (PRISMA-P) 2015: elaboration and explanation. BMJ 2015;349:g7647.

31. Stroup DF, Berlin JA, Morton SC, et al. Meta-analysis of observational studies in epidemiology: a proposal for reporting. Meta-analysis Of Observational Studies in Epidemiology (MOOSE) group. JAMA 2000;283:2008-12.

32. Montgomery SA, Asberg M. A new depression scale designed to be sensitive to change. Br J Psychiatry 1979;134:382-9.

33. Hamilton M. A rating scale for depression. J Neurol Neurosurg Psychiatry 1960;23:56-62.

34. Posner K, Brown GK, Stanley B, et al. The Columbia-Suicide Severity Rating Scale: initial validity and internal consistency findings from three multisite studies with adolescents and adults. Am J Psychiatry 2011;168:1266-77.

35. Wells GA, Shea B, O'Connell D, et al; The Newcastle-Ottawa Scale (NOS) for assessing the quality of non-randomised studies in metaanalysis. Ottawa, Ontario: Ottawa Health Research Institute, 2004.

36. von Elm E, Altman DG, Egger $\mathrm{M}$, et al. The Strengthening the Reporting of Observational Studies in Epidemiology (STROBE) statement: guidelines for reporting observational studies. Lancet 2007;370:1453-7.

37. Guyatt GH, Oxman AD, Vist GE, et al. GRADE: an emerging consensus on rating quality of evidence and strength of recommendations. BMJ 2008;336:924-6.

38. Higgins JPT, Green S, eds. Cochrane Handbook for Systematic Reviews of Interventions. Version 5.1.0. Updated, 2011.

39. Egger M, Smith GD, Phillips AN. Meta-analysis: principles and procedures. BMJ 1997;315:1533-7.

40. Emanuel EJ, Onwuteaka-Philipsen BD, Urwin JW, et al. Attitudes and Practices of Euthanasia and Physician-Assisted Suicide in the United States, Canada, and Europe. JAMA 2016;316:79-90. 